\title{
Modelos educativos a distancia ligados a los desarrollos tecnológicos
}

\author{
Lorenzo García Aretio \\ Miriam García Blanco \\ Universidad Nacional de Educación a Distancia, UNED
}

Recibido: 16 septiembre 2015 / Aceptado: 29 febrero 2016

ISSN: $1697-7467$

\begin{abstract}
RESUMEN: Diferentes factores de carácter sociopolítico, económico y psicopedagógico vinieron influyendo para la emergencia de propuestas educativas que se permitían prescindir de la sistemática relación cara a cara entre docentes y estudiantes. La evolución de estos sistemas corrió pareja al desarrollo tecnológico de nuestra sociedad. Esa evolución generó diferentes modelos de enseñanza a distancia que vinieron propiciando aprendizajes de calidad básicamente comparables a las tradicionales formas de enseñar y aprender.

Para ello, se revisan en el artículo formas de adquirir conocimientos, diferentes a los formatos presenciales y soportadas en tecnologías diversas. Modelos diferentes que han ido significándose a través de estos últimos años. Tras los modelos más convencionales de educación a distancia, basados en el medio impreso, el teléfono y los recursos audiovisuales, ahí aparecen denominaciones tales como el e-le arning, blended-learning, aprendizaje colaborativo en red, aprendizaje móvil, ubicuo, etc. En fin, aprendizajes mediados que vienen culminando estos años con la irrupción de los MOOC. Estas formas diferentes, no presenciales, de enseñar y aprender se vienen aplicando con éxito desde hace años, asegurando su calidad, siempre que se doten de las bases y principios pedagógicos adaptados en nuestros días a las posibilidades que nos brinda la sociedad digital.

Palabras clave: educación a distancia, tecnología de la educación, modelos didácticos, innovación pedagógica.
\end{abstract}

\section{Distance Education Models Linked to Technological Developments}

ABSTRACT: Different socio-political, economic and psycho-pedagogical factors influenced the emergence of educational proposals that could dispense with the systematic face to face relationship between teachers and students. The evolution of these systems ran even to the technological development of our society. These developments led to different models of distance learning that fostered quality learning basically comparable to traditional forms of teaching and learning.

In this context, ways of acquiring knowledge supported by diverse technologies and different from the classroom are reviewed. Different models that have come to stand out in recent years. After the more conventional models of distance education, based on the print medium, the telephone and audiovisual resources, there appear names such as e-learning, blended learning, collaborative learning network, mobile learning, ubiquitous, etc. In summary, mediated learning that has culminated in recent years with the emergence of the MOOC. These different, non-contact, teaching and learning methods have been applied successfully for years, ensuring its quality, as long as they are provided with the bases and pedagog1lical principles adapted today to the possibilities offered by the digital society.

Keywords: distance education, education technology, didactic models, pedagogical innovation. 


\section{INTRODUCCIÓN}

A lo largo de la historia reciente, último siglo y medio, han ido apareciendo propuestas educativas a distancia que han venido configurando formas, escenarios, esquemas o modelos más o menos consolidados tomando como referente a las instituciones, a los programas ofrecidos, a las metodologías pedagógicas o a los medios y recursos utilizados. De ahí han surgido diferentes modelos, tipologías o variantes. En concreto:

- instituciones unimodales (sólo de educación a distancia) o bimodales o mixtas;

- modelos de aprendizaje unimodal o semipresencial;

- $\quad$ propuestas centradas en el alumno, en el docente, en los materiales o en la tecnología;

- $\quad$ según la vocación institucional, con más o menos énfasis en la interinstitucionalidad o internacionalidad de los programas, de los docentes y de los propios estudiantes;

- modelos de docencia organizados según los grados y modos de interacción síncrona o asíncrona, vertical u horizontal;

- modelos centrados en las teorías de aprendizaje aplicadas en entornos no presenciales;

- modelos basados en la evolución o uso de determinados medios o recursos (impresos, audiovisuales, digitales, etc.).

En el presente trabajo hemos optado por centrar nuestra atención sobre esos últimos escenarios o modelos, surgidos en función de la integración de los diferentes medios y recursos que el desarrollo tecnológico ha venido favoreciendo. Diversas circunstancias a lo largo de la historia provocaron la búsqueda de otras soluciones y formas diferentes de enseñar y aprender. Los sistemas presenciales no se veían capaces de atender los requerimientos propios que el progreso social venía exigiendo (García Aretio, 2002). Así, entre otros factores impulsores de aquella época, podríamos destacar:

- Las estructuras rígidas y anquilosadas de la Universidad y el aumento de demanda social de educación. Era necesario arbitrar otras vías y modos alternativos de enseñar y aprender (Wedemeyer, 1981) que superasen las necesidades espaciotemporales propias de los sistemas educativos convencionales (García Aretio, 2002; Verdú, 1998; Menon, 1998).

- La existencia de amplias capas sociales alejadas de la educación, pero con motivación y capacidad suficientes como para acometer estudios superiores (Holmberg, 1989; Kaye y Rumble, 1979; Wedemeyer, 1981; Moore y Kearsley, 1996; Keegan, 1980; García Aretio, 2002).

- La necesidad de educación permanente, como rasgo definitorio de la sociedad actual (Delors, 1996). Se comenzaban a exigir otras formas de aprendizaje que no requiriesen de la presencia sistemática de educador y alumno en tiempo y espacio determinados (Edwards, Ranson y Strain, 2010).

- Los elevados costes de la educación superior (Nelson y Hevert, 2006) y la rentabilidad de los estudios a distancia que vino siendo avalada por diferentes trabajos (Pascual, 2003; Wagner, 1977; Mace, 1978; Snowden y Daniel, 1980; Rumble, 1997).

Los factores señalados podríamos aplicarlos hoy al tema objeto de este monográfico, la enseñanza de las lenguas extranjeras. Desde los tiempos en que se dio carta de naturaleza a la educación a distancia, hasta las recientes experiencias con los MOOC, el aprendizaje y 
mejora del conocimiento de los diferentes idiomas han formado parte de cursos concretos en modalidades no presenciales. Conforme las tecnologías han ido penetrando los ámbitos educativos, han proporcionado medios para ayudar a reestructurar tanto los contenidos como los procesos de enseñanza de idiomas.

El método que seguiremos para concretar nuestro trabajo será el de realizar un recorrido histórico (García Aretio, 1999) por las grandes etapas, marcadas por el desarrollo tecnológico, que dieron carta de naturaleza a la educación a distancia en cualesquiera de sus diferentes presentaciones y formatos.

\section{VARIANTES, ESCENARIOS Y MODELOS EN SISTEMAS NO PRESENCIALES}

\subsection{Los modelos basados en materiales impresos}

En 1840, Isaac Pitman en Inglaterra programó un sistema de taquigrafía a base de tarjetas e intercambio postal con los alumnos (Homberg, 2005), que pudo suponer el inicio de la educación a distancia en su formato de enseñanza por correspondencia, y en 1873 Anna E.Tickner fundó en Boston la Society for the Promotion of Study at Home (Lambert, 1983). Poco después, destacar que el correo postal como medio de comunicación en la enseñanza superior por correspondencia, se empleó en el Chautauqua Correspondence College fundado en 1881 (Bittner y Mallory, 1933). Así se comenzaban a vislumbrar las posibilidades de la comunicación asíncrona en estos modelos educativos. En 1856 el francés Charles Toussaint, que ya enseñaba su lengua en Alemania, y Gustav Langenscheidt miembro de la Sociedad de Lenguas Modernas de Berlín, impartieron probablemente las primeras clases con material diseñado para el autoestudio fundando el primer instituto para enseñar lenguas extranjeras por correspondencia (Noffsinger, 1926).

En esta primera andadura, el modelo era simple y potenciado gracias al desarrollo del correo postal y de la imprenta: textos muy rudimentarios sin especificaciones didácticas, sucesivamente mejorados para animar al estudio independiente. La comunicación entre formadores y estudiantes progresivamente se fue afianzando mediante un formato textual, manuscrito y asíncrono. Sin embargo ésta supuso una etapa que ha permanecido muchas décadas, existiendo experiencias cercanas en el tiempo que han seguido y continúan utilizando el material impreso como sustento fundamental de los programas a distancia, junto al correo postal. En algunas partes del mundo, fundamentalmente de África y Asia, el medio impreso continúa siendo un recurso sustancial en este tipo de programas, sobre todo en la formación de docentes (Burns, 2011). Como apoyo comunicativo síncrono a los estudiantes, que complementaba el que se venía realizando a través del correo postal, reseñamos el teléfono, sin duda el más personalizado de los existentes hasta el advenimiento de Internet (Garrison, 1985). Estábamos ante la primera posibilidad de comunicación personal y síncrona entre profesores y alumnos.

Este modelo iniciático de la educación a distancia, continuó ofreciéndose durante décadas, incluso con el advenimiento de tecnologías más avanzadas que favorecían una metodología basada en texto escrito, pero a través de Internet o, anteriormente, mediante el soporte CDROM o DVD, en formatos tipo pdf (Latchem y Jung, 2010). Y cuando los diseños de esos 
materiales, acompañados de las correspondientes vías de comunicación eran pertinentes, la calidad de aquellos programas era, y continúa siendo, reconocida (Perraton, 1993) y mucho más cuando a ello se sumaba el correspondiente apoyo humano en forma de tutorías, fuesen éstas presenciales o a través del correo o el teléfono (Hord, Rutherford, HulingAustin y Hall, 2006). No olvidemos que esta educación a distancia soportada fundamentalmente en textos impresos, correo postal y teléfono fue la que inicialmente dio soporte a las nacientes universidades unimodales (enseñanza sólo a distancia), década de los años 70 del pasado siglo, al hilo de la creación de la Open University Británica en 1969.

\subsection{Con la radio y el audio}

Receptores de radio, reproductores audiocasetes o reproductores de CD o DVD fueron y son dispositivos de sencillo uso y relativamente económicos. Las primeras licencias de radio a las universidades norteamericanas se otorgaron a partir de 1921 a las de Utah, Wisconsin y Minnesota (Casey, 2008). A ellas siguieron otras experiencias que vinieron a potenciar las posibilidades de este medio como recursos educativo en general y para la enseñanza a distancia y de idiomas, en particular. Posibilidades que se acrecentaron con la irrupción de la radio de transistores ( $\sin$ cables), un incipiente m-learning. En la década de los 60 del siglo pasado esas posibilidades fueron multiplicadas con el nacimiento de los audio-casetes que inicialmente se usaban principalmente como guías y motivación para el estudio de los materiales impresos (Laaser, 1986) y posteriormente tuvieron relevancia en materias específicas como el estudio de idiomas. La radio tiene un gran potencial en términos de apoyo a los estudiantes que están aprendiendo a distancia (Bansal y Choudhary, 1999) que puede ofrecer enseñanza de calidad a poblaciones dispersas y, además, a un bajo costo por unidad de producción (Chandar y Sharma, 2003).

La radio digital, a través de la Web se viene utilizando hoy, a veces simultáneamente a la radio convencional. Las emisiones digitales aportan indudables ventajas dada la escasa inversión que precisa emitir radio a través de Internet. Amén de las posibilidades de complementar con otros recursos multimedia (Gaible y Burns, 2007). En función de lo que interesa en este monográfico, un buen ejemplo de aprendizaje de inglés por parte de profesores y estudiantes es el de South Africa's Open Learning Systems Educational Trust (OLSET) "English in Action". Desde 1993 hasta 2009, 52.000 docentes y unos dos millones de estudiantes se formaron para mejorar su inglés oral y escrito (Potter y Naidoo, 2009).

La radio ha servido en diferentes experiencias y proyectos, como recurso sustancial de enseñanza y diversos proyectos de alfabetización a distancia (Ofulue, 2011). Es el caso, en España, de Radio ECCA (Emisora Cultural de Canarias), nacida en 1965, y que aún continúa en nuestros días con su tarea. Experiencias similares gozan de buena acogida en países nórdicos de Europa (Perona y Barbeito, 2007) y también en América Latina (García Aretio, 1999) en todos los casos solían estar presentas iniciativas referidas al aprendizaje de idiomas.

\subsection{Con la televisión y el vídeo}

Las expectativas educacionales de la televisión fueron muy elevadas inicialmente (Westera, 2012). Destaquemos que en 1956 arranca la emisión de programas educativos por 
televisión el Chicago TV College cuya influencia se dejó notar pronto en otras universidades norteamericanas que no tardaron en crear unidades de enseñanza a distancia basadas en la televisión. Es de destacar que en 1960 se funda en China el Beijing Broadcasting and Television. La imagen del docente, siempre clave en los sistemas presenciales, se hace ahora complemento en las propuestas a distancia, gracias a las tecnologías de imagen, cine, vídeo, televisión, videoconferencia, etc. (Peters, 2000). La televisión en sus diferentes soportes, al mostrar situaciones reales o simuladas muy cercanas a la realidad, puede suplir e incluso superar a la propia experimentación, anticipando resultados, permitiendo visualizar procesos y procedimientos y aumentar la motivación a través del estímulo audiovisual (Gaible y Burns, 2007; Saltrick, Honey, y Pasnik, 2004). En este sentido han sido muchas las experiencias en la utilización de la televisión como soporte sustancial en programas de educación a distancia (Burns, 2011).

Por su parte el vídeo, sea en el formato que fuere, añade todas las ventajas de la televisión, con las agregadas de la autonomía del usuario tanto en la situación geográfica donde puede activarse, como en las posibilidades de repetición de contenidos o fracción de los mismos. Los ancho de banda actuales, el cable, la fibra óptica, etc., nos han mostrado trabajos de experiencias educativas con videos en línea que ponen de manifiesto que los alumnos están entusiasmados con esa posibilidad (Veeramani y Bradley, 2008; Woo et al, 2008) prefiriendo videos a textos académico (Chan, 2010, Donkor, 2011). Textos soportados en línea que insertan vídeos están aumentando en uso y eficacia en la educación universitaria (VitalSource, 2014). En este mismo ámbito, referido al recurso audiovisual, podemos ubicar las videoconferencias, las videoclases y todo tipo de grabaciones audiovisuales utilizadas con finalidades pedagógicas (Vázquez, Fombona y Fernández, 2013).

\subsection{Los modelos basados en Internet}

Desde la década de los años 70 del siglo pasado con el nacimiento del correo electrónico y de las listas de distribución se iniciaron experiencias de aprendizaje en línea que se consolidaron hacia finales del siglo una vez se va desarrollando a gran velocidad la WWW y van experimentándose plataformas virtuales o sistemas de gestión de aprendizaje (LMS - Learning Management System) en los que, además de los contenidos, se gestionan los sistemas de comunicación, el seguimiento de la gestión, la instrucción personalizada, los procesos de evaluación, etc. (Watson y Watson, 2007). Estamos ante un nuevo enfoque de la enseñanza-aprendizaje que se soporta en medios y dispositivos electrónicos como herramientas para tratar de mejorar los procesos de formación, comunicación e interacción (Sangrá, Vlachopoulos y Cabrera, 2012). Estaríamos refiriéndonos a una forma evolucionada de educación a distancia en la que la mediación se lleva a cabo a través de medios y dispositivos digitales con las ventajas que conlleva la rapidez y eficacia en todo el proceso. Cierto que estas formas de enseñar y aprender, fundamentalmente a través de plataformas digitales (LMS), han acogido cursos de todo tipo de saberes y, sin duda, en casi todos los casos, de aprendizaje de lenguas extranjeras, dadas las ventajas de los formatos multimedia en texto, audio y vídeo y las posibilidades de interacción vertical (docente-estudiante) y horizontal (entre pares).

Posteriormente llegamos al concepto de Web 2.0 (O’Reilly, 2007) que más que una tecnología se configuró como una actitud (Davis, 2005 y Wesch, 2009). Las posibilidades 
de interacción que ofrece la red, más aún en su imagen de web 2.0, abrió posibilidades para alumbrar nuevas experiencias de enseñanza y aprendizaje. Las comunicaciones síncronas, asíncronas, verticales y horizontales, utilizadas con sentido pedagógico apropiado, ofrecen modelos alternativos claros y eficaces a los sistemas presenciales de enseñanza en general y de lenguas extranjeras en particular, a través de las numerosas herramientas 2.0, tales como blogs, wikis, RSS, podcast, redes sociales, intercambio de ficheros de texto, audio y vídeo p2p, etc. La sociabilidad que propicia la Web 2.0 supone un potencial de gran envergadura para la educación y una posibilidad increíble para la educación a distancia (Boyd, 2007). Colaboración, socialización y aumento de la eficiencia pedagógica pero a la vez, también, mayor independencia y autonomía para el estudiante (Franklin y Harmelen, 2007) y más altos grados de satisfacción (Barkley, Cross y Major, 2005). La apertura de sistemas de aprendizaje basados en la Web 2.0 contrastó con los entornos más cerrados, propios de los LMS o plataformas virtuales.

\subsection{El blended learning}

El blended learning entendido como aquel modo de aprender que combina la enseñanza presencial con la modalidad a distancia (Marsch, Mcfadden y Price, 2003; Bersin, 2004), puede ofrecer resultados de calidad, por encima de los cursos presenciales y de los exclusivamente en línea (Means y otros, 2009; Garrison y Vaughan, 2008). Esta modalidad parece apropiada para la mayoría de estilos de aprendizaje (Wakefield, Carlisle, Hall, y Attree, 2008). También hoy se vienen ofreciendo MOOC híbridos (Anders, 2015).

En los últimos años, muy cercano a estos conceptos viene emergiendo con fuerza el movimiento Flipped Classroom, entendido como aquella técnica educativa que contempla dos escenarios: actividades interactivas de aprendizaje en grupo dentro del aula y aprendizaje individual a través de los recursos digitales, fuera de la clase (Bishop y Verleger (2013). Combinación, al fin y al cabo, de las dos modalidades.

\subsection{El m-learning y la nube}

Ya existen más móviles que humanos (Oliver, 2015). Las posibilidades de personalización de los dispositivos móviles, la superación de las limitaciones espaciotemporales, las posibilidades de emisión, grabación y recepción de texto, imágenes, audio y vídeo, sean síncronas o asíncronas, a través de estas tecnologías, suponen un puntal relevante de la nueva educación a distancia (Keskin y Metcalf, 2011). Nos referimos a los teléfonos inteligentes, tabletas, consolas, ordenadores portátiles, etc., que cuentan con conectividad fuera de las habituales conexiones fijas o de wifi restringido (Dhir, Gahwaji y Nyman, 2013). El acceso a las fuentes del conocimiento, el aprendizaje, es posible así en cualquier momento y desde cualquier lugar (ubicuidad) (Burbules, 2012) e, incluso, en movimiento. El debate educativo de hoy se ocupa de estos dispositivos móviles como instrumentos eficaces para el aprendizaje (Cabot, Garcia-Lopez, de-Marcos y Abraham, 2014) y se muestran como una gran oportunidad para la nueva educación a distancia (Brown y Mbati, 2015). El aprendizaje de lenguas extranjeras, la traducción textual y oral simultánea, la interactividad social, la sensibilidad al contexto, la investigación y la innovación a través de dispositivos móviles nos disponen para nuevos enfoques educativos (Crompton, 2013; Liu y Chen, 2015). 
La cantidad y economía de aplicaciones disponibles en entornos móviles (Pareja, Calle y Pomposo, 2016; Ibáñez, Jordano y Vermeulen, 2016; Read, y Barcena, 2016), que se vienen evaluando con rigor (Santiago, 2012; Arús, Rodríguez y Calle, 2013; Munday, 2016) ofrecen posibilidades educativas innovadoras. La utilización de los SMS y el chat en móviles para propósitos educativos es uno método sencillo de interactividad de calidad (Hayati, Jalilifar y Mashhadi, 2013; Castrillo, Bárcena y Martín, 2014). Por su parte, el valor añadido del juego y el entretenimiento para aprender idiomas mediante dispositivos móviles (Sandberg, Maris y Hoogendoom, 2014; Rico, y Agudo, 2016) se suman a las nuevas corrientes de gamificación en entornos educativos. Las posibilidades de la realidad aumentada aplicada a la enseñanza de idiomas (Hockly y Dudeney, 2014), también sugieren nuevas oportunidades de aprendizaje en cualquier lugar y momento.

Si sumamos los dispositivos móviles al almacenamiento en la nube, las experiencias de aprendizaje pueden facilitar un gran avance para el desarrollo futuro de la educación (Hirsch y Ng, 2011). La nube supera ciertas limitaciones con las que cuentan los dispositivos móviles con respecto a la capacidad de procesamiento y de memoria, el alto coste de las tarifas de conexión y la lentitud de la red en muchos casos (Li, 2010; Kitanov y Davcev, 2012).

\subsection{Los MOOC}

Sin duda el fenómeno más destacado en el ámbito educativo de masas en nuestra sociedad digital, ha sido el de los Cursos Masivos Abiertos y a Distancia (MOOC - Massive, Open, Online Courses). Fenómeno calificado por Brook (2012) como el tsunami de los campus. Ese mismo año 2012 (Pappano, 2012) fue calificado como el año de los MOOC. Si bien, pronto se pasó de la máxima expectación a la frustración de algunos. Los MOOC, ni son la solución a todos los males educativos del momento, pero tampoco la trampa o el mero negocio. Ni tan excelentes, ni tan deplorables. La propia investigación los va poniendo en su lugar. Es cierto que a lo largo de 2015 los MOOC han seguido ahí, no como se soñaron al inicio, pero el hecho de que desde 2011 millones de personas se han inscrito en un MOOC certifica que estamos ante un fenómeno que requiere la atención debida (García Aretio, 2015).

Es de notar que la enseñanza de lenguas extranjeras viene estando presente en la mayoría de propuestas de aquellas instituciones que se han decidido apostar por el modelo MOOC, con sus múltiples matices e interpretaciones que, en ningún caso deberían obviar la perspectiva pedagógica y visión desde la educación (Cabero, 2015).

Es numerosa, variada y actual la literatura científica surgida en torno a este fenómeno, por ejemplo, la referida a las diferentes clasificaciones y tipologías de MOOC, más allá de las clásicas y originales de los cMOOC (conectivistas) y xMOOC (más comerciales) (Cabero, Llorente y Vázquez, 2014); la que hace mención a la figura y tipología de docentes, facilitadores o asesores en estos cursos (Marauri, 2014); las inmensas posibilidades referida a la conformación de la participación y creación de comunidades de aprendizaje (Torres y Gago, 2014). El debate sobre las oportunidades y amenazas que pueden suponer para las universidades es vibrante (Capdevilla y Aranzadi, 2014); su relación o ¿dependencia? de los recursos educativos abiertos también importa (Tovar y Lesko, 2014). Otro tema también crítico es el referido a la evaluación, valoración y gestión de los MOOC (Aguaded y Medina, 2015; Baldomero, 2015; Mengual, Roig y Lloret, 2015); el de la eficacia o no sobre los aprendizajes adquiridos y el nivel de satisfacción de los participantes (Castaño, Maiz 
y Garay, 2015; Shin, y Kang, 2015), así como aquellos trabajos referidos a los aspectos financieros que implican (Hollands y Tirthali, 2014).

Para terminar el recorrido, somos conscientes de que la evolución tecnológica ligada a las innovaciones pedagógicas nos obligan a citar, aunque sea brevemente, otras propuestas que también podrían aplicarse al estudio de idiomas. Así, la mecánica del juego aplicada a efectos educativos, léase gamificación (gamification) (Simões, Redondo, y Vilas, 2012), tiene algunas posibilidades también interesantes en el mundo del aprendizaje de lenguas. Igualmente, la realidad aumentada ligada a los aprendizajes móviles, nos ofrecen nuevos posibles enfoques pedagógicos (Bower et al, 2014).

\section{Conclusión}

Puede resultar simplista hablar de metodologías a distancia si no se realiza el esfuerzo de delimitar la propuesta correspondiente que, además de lo señalado en este trabajo, habría que concretarlo según institución y país. Las variantes son innumerables, aunque tratando de perfilar modelos que a lo largo de la corta historia de la educación a distancia han venido marcando la modalidad, podríamos señalar los siguientes:

- Enseñanza/aprendizaje por correspondencia, basados en textos impresos como soporte de los contenidos y en una interacción básicamente a través del correo postal y poco después, del teléfono.

- Enseñanza/aprendizaje a distancia con apoyos audiovisuales, entre los que vinieron destacando la radio, el audio, la televisión, el vídeo y los sistemas de videoconferencia.

- Enseñanza/aprendizaje a distancia en entornos virtuales, con dos perspectivas cronológicas, primero enfoques en línea con escasa apertura, restringidos y bastante cerrados que se cifraron en las plataformas virtuales o LMS y, en segundo lugar, con una dimensión más abierta, los espacios interactivos de aprendizaje a través de las tecnologías de la web 2.0.

- Enseñanza/aprendizaje semipresencial (b-learning). A caballo de los avances tecnológicos y tratando de hacer confluir los aspectos más positivos de los modelos presenciales y virtuales, mezclándolos adecuadamente.

- Enseñanza/aprendizaje móvil (m-learning) soportados en los dispositivos móviles que ofrecen gran autonomía espacio temporal y que con el apoyo del almacenamiento en la nube, viene suponiendo un tipo de aprendizaje ubicuo (u-learning).

- MOOC. Los cursos masivos, abiertos y en línea como último aporte dentro de los sistemas a distancia que ofrecen posibilidades en la democratización del acceso a la educación mediante una oferta de calidad docente e institucional.

Modelos todos ellos que con diferente énfasis podemos encontrar en diversas zonas de nuestro universo, marcadas por sus correspondientes desarrollos socioeconómicos y también por las distintas necesidades de formación, sobre todo de la población adulta, que sin duda hoy en los países desarrollados, a través de los entornos virtuales, se pueden aprovechar de escenarios altamente vigorosos para la enseñanza y el aprendizaje de idiomas dado que potencian las diferentes metodologías que en entornos presenciales ya se mostraron como eficaces. 
Concluimos señalando que cuando los diseños de cualesquiera de las propuestas y modelos de educación a distancia aquí esbozadas, se realizan atendiendo a los principios y bases pedagógicos que iluminan a esta modalidad, la eficacia, la calidad de las mismas, está asegurada tal y como se reseña en multitud de trabajos científicos (García Aretio y Ruíz, 2010) que certifican calidades al menos similares a las de los procesos educativos presenciales.

\section{Bibliografía}

Aguaded, I., y Medina, R. (2015). "Criterios de calidad para la valoración y gestión de MOOC". En RIED. Revista Iberoamericana de Educación a Distancia, 18, 2: 119-143.

Anders, A. (2015). "Theories and Applications of Massive Online Open Courses (MOOCs) : The Case for Hybrid Design". En The International Review Of Research In Open And Distributed Learning, 16, 6.

Arús, J., Rodríguez, P., y Calle, C. (2013). "A pedagogic assessment of mobile learning applications". In Proceedings of ICDE 2013: Mobilizing Distance Education, 630-643.

Baldomero, M. (2015). "La valoración de MOOC: Una perspectiva de calidad". En RIED. Revista Iberoamericana de Educación a Distancia, 18, 2: 171- 195.

Bansal, K., y Choudhary, S. S. (1999). "Interactive Radio for Supporting Distance Education: An evaluative study", en Indian Journal of Open Learning, 8, 1: 61 - 71.

Barkley, E. F.; Cross, K. P. y Major, C. H. (2005) Collaborative learning techniques: A handbook for college faculty. San Francisco: Jossey-Bass.

Bersin, J. (2004). The blended learning book: Best practices, proven methodologies, and lessons learned. San Francisco, CA: Pfeiffer

Bishop, J.L., y Verleger, M.A. (2013). "The flipped classroom: A survey of the research", en ASEE National Conference Proceedings, Atlanta, GA, disponible en: http://goo.gl/mVH6zd, consultado en 2 de septiembre de 2015.

Bittner, W.S. y Mallory, H.F. (1933). University Teaching by Mail. New York: Mcmillan.

Boyd, D. M. (2007) “The significance of social software”. En Burg, T. N. y Schimidt, J. (eds.). BlogTalks reloaded: Social software research \& cases, Norderstedt, disponible en: http:// goo.gl/Y6c1MX, consultado el 9 de agosto de 2015.

Bower, M., Howe, C., McCredie, N., Robinson, A. y Grover, D. (2014). “Augmented Reality in education - cases, places and potentials", en Educational Media International, 51, 1: 1-15.

Brown, T., y Mbati, L. (2015)." Mobile learning: Moving past the myths and embracing the opportunities". En The International Review Of Research In Open And Distributed Learning, 16, 2.

Burbules, N. (2012). "Entrevista a Nicolás Burbulés", en Webinar, 2012 disponible en: http://goo. gl/DpJEcH, consultado el 11 de mayo de 2015.

Burns, M. (2011). Distance Education for Teacher Training: Modes, Models and Methods. Waltham: Education Development Center.

Cabero, J. (2015). "Visiones educativas sobre los MOOC". En RIED. Revista Iberoamericana de Educación a Distancia, 18, 2: 39-60.

Cabero, J., Llorente, M. C., y Vázquez, A. I. (2014). "Las tipologías de MOOC: su diseño e implicaciones educativas". Profesorado. En Revista de Curriculum y formación del profesorado, 18, 1: 14-26.

Cabot, A. G., Garcia-Lopez, E., de-Marcos, L., y Abraham-Curto, J. (2014). "Adapting learning contents to mobile devices and context to improve students' learning performance: A case study". Journal of Universal Computer Science, 20, 15: 2032-2042. 
Capdevilla, R., y Aranzadi, P. (2014). "Los Cursos Online Masivos y Abiertos: ¿Oportunidad o amenaza para las universidades iberoamericanas?", en RIED. Revista Iberoamericana de Educación a Distancia, 17, 1: 69-82.

Casey, D. M. (2008). "A Journey to Legitimacy: The Historical Development of Distance Education through Technology", en TechTrends, 52, 2: 45-51.

Castaño, C.; Maiz, I., y Garay, U. (2015). Percepción de los participantes sobre el aprendizaje en un MOOC. En RIED. Revista Iberoamericana de Educación a Distancia, 18, 2: 197-221.

Castrillo de Larreta-Azelain, MD, Martin-Monje, E y Bárcena, E. (2014). "New Forms of Negotiating Meaning on the Move: the Use of Mobile- Based Chatting for Foreign Language Distance Learning". En IADIS International Journal on WWW/Internet Vol. 12, No. 2, pp. pp. 51-67.

Chandar, U. y Sharma, R. (2003). "Bridges to Effective Learning Through Radio", en IRRODL, 4, 1.

Chan, Y. M. (2010). "Video instructions as support for beyond classroom learning", en Procedia Social and Behavioral Sciences, 9: 1313-1318.

Crompton, H. (2013). "A historical overview of m-learning: Toward learner-centered education", en Z. L. Berge y L. Y. Muilenburg (Eds.) Handbook of mobile learning. New York: Routledge Taylor and Francis Group.

Davis, I. (2005). Talis, Web 2.0 and All That, available, disponible en: http://goo.gl/BMz9bJ, consultado el 17 de julio de 2015.

Delors, J. (1996): La educación encierra un tesoro. Informe a la UNESCO de la Comisión Internacional sobre la educación para el siglo XXI. Madrid: Santillana.

Dhir, A., Gahwaji, N. M., y Nyman, G. (2013). "The role of the iPad in the hands of the learner". En Journal of Universal Computer Science, 19, 5: 706-727.

Donkor, F. (2011). "Assessment of learner acceptance and satisfaction with video-based instructional materials for teaching practical skills at a distance". In IRRODL, 12, 5.

Edwards, R.; Ranson, S. y Strain, M. (2010). "Reflexivity: towards a theory of lifelong learning" en International Journal of Lifelong Education, 21, 6.

Faure et. al. (1973). Aprender a ser. La educación del futuro. Madrid: Alianza.

Franklin, T. y Harmelen, M. (2007). Web 2.0 for Learning and Teaching in Higher Education. "London: The Observatory of Borderless Higher Education", disponible en: http://goo.gl/ dG2nia, consultado el 17 de Julio de 2015.

Gaible, E., y Burns, M. (2007). Using technology to train teachers: Appropriate uses of ICTs for professional development. Washington, DC: World Bank, disponible en http://goo.gl/ LounbS, consultado el 17 de julio de 2015.

García Aretio, L. (1999). "Historia de la educación a distancia", en RIED. Revista Iberoamericana de Educación a Distancia, 2, 1: 11-40.

García Aretio, L. (2002). Educación a distancia. De la teoría a la práctica. Madrid: Ariel.

García Aretio, L. (2011). "Perspectivas teóricas de la educación a distancia y virtual", en Revista Española de Pedagogía, 249: 255.

García Aretio, L. (2014). Bases, mediaciones y futuro de la educación a distancia en la sociedad digital. Madrid: Síntesis.

García Aretio, L. (2015). "MOOC: ¿tsunami, revolución o moda pasajera?”, en RIED. Revista Iberoamericana de Educación a Distancia, 18, 1.

García Aretio, L. y Ruíz Corbella, M. (2010) "La eficacia de la educación a distancia: ¿un problema resuelto?", en Teoría de la Educación, 22, 1: 141-162.

Garrison, G. R. (1985). "Three Generations of Technological Innovation”, en Distance Education, 6, 2: $235-241$. 
Garrison, D. M., \& Vaughan, N. D. (2008). Blended learning in higher education: Framework, principles, and guidelines. San Francisco, CA: Jossey-Bass.

Hayati, A. Jalilifar, A., y Mashhadi, A. (2013). "Using short message service (SMS) to teach English idioms to EFL students". En British Journal of Educational Technology, 44, 1: 66-81.

Hirsch, B., y Ng, J. W. (2011). "Education beyond the cloud: Anytime-anywhere learning in a smart campus environment", en Internet Technology and Secured Transactions (ICITST), 2011 International Conference. IEEE: 718-723.

Hockly, N., y Dudeney, G. (2014). Going mobile teaching with handheld devices. En Surrey: Delta Publishing.

Hollands, F., y Tirthali, D. (2014). "Resource requirements and costs of developing and delivering MOOCs". En The International Review Of Research In Open And Distributed Learning, 15, 5.

Holmberg, B. (1989). Theory and practice of distance education. New York: Toutledge.

Holmberg, B. (2005). The Evolution, Principles and Practices of Distance Education. Oldenburg: University of Oldenburg

Hord, S., Rutherford, W. L., Huling-Austin, L., y Hall, G. E. (2006). Taking charge of change. Austin, TX: Southwest Educational Development Laboratory.

Ibáñez, A., Jordano, M., y Vermeulen, A. (2016). "Diseño y evaluación de VISP, una aplicación móvil para la práctica de la competencia oral". En RIED. Revista Iberoamericana de Educación a Distancia, 19, 1: 63-81.

Kaye, A. y Rumble, G. (1979). Analysing distance learning systems. London: Open University.

Keegan, D. J. (1980). On the nature of distance education. Hagen: ZIFF.

Keskin, N.O. y Metcalf, D. (2011). "The current perspectives, theories and practices of mobile learning", en The Turkish Online Journal of Educational Technology, 10, 2: 202-208

Kitanov, S., y Davcev, D. (2012). "Mobile cloud computing environment as a support for mobile learning", en Cloud Computing 2012, The Third International Conference on cloud computing, GRIDs, and Virtualization, pp. 99-105.

Laaser, W. (1986). "Some Didactic Aspects of Audio-Cassettes in Distance Education", en Distance Education, 7, 1: 143-52.

Lambert, M. P. (1983). "New course planning. The strategy and tactics of developing a home study course", en NHSC News. Washington: National Home Study Council.

Latchem, C., y Jung, I. (2010). Distance and blended learning in Asia. New York, NY: Routledge.

Lengrand, P. (1973): Introducción a la educación permanente. Barcelona: Teide

Li, J. (2010). "Study on the development of mobile learning promoted by cloud computing", en IEEE 2010 2nd International Conference on Information Engineering and Computer Science (ICIECS), pp. 1-4.

Liu, P., y Chen, C. (2015). "Learning English through actions: A study of mobile-assisted language learning”. En Interactive Learning Environments, 23, 2: 158-171.

Mace, J. (1978). "Mythology in the making; is the Open University really cost-effective?", en Higher Education, 7: 295-309.

Marauri, P. M. (2014). "La figura de los facilitadores en los Cursos Online Masivos y Abiertos (COMA / MOOC): nuevo rol profesional para los entornos educativos en abierto". En RIED. Revista Iberoamericana de Educación a Distancia, 17, 1: 35-67.

Marsch, G. E.; Mcfadden, A. C. y Price, B. (2003). "Blended Instruction: Adapting Conventional Instruction for Large Classes", en Online Journal of Distance Learning Administration, VI, IV.

Mengual, S., Roig, R., y Lloret, C. (2015). "Validación del Cuestionario de evaluación de la calidad de cursos virtuales adaptado a MOOC". En RIED. Revista Iberoamericana de Educación a Distancia, 18, 2: 145-169. 
Means y otros (2009). Evaluation of Evidence-Based Practices in Online Learning: A Meta-Analysis and Review of Online Learning Studies. Washington: U.S.: Department of Education.

Menon, M.E. (1998). "Factors influencing the demand for higher education: The case of Cyprus", en Higher Education, 35, 3.

Moore, M. G. y Kearsley, G. (1996). Distance Education: As systems view. Belmont, CA: Wadswort.

Munday, P. (2016). "The case for using DUOLINGO as part of the language classroom experience". En RIED. Revista Iberoamericana de Educación a Distancia, 19, 1: 83-101.

Nelson, R. y Hervert, K. (2006). "Effect of class size on economies of scale and marginal costs in higher education", en Applied Economics, 24, 5.

Noffsinger, J. S. (1926). Correspondence Scholls, Lyceums, hautauquas. New York: Macmillan.

Ofulue, C.I. (2011). "Literacy at a Distance in Multilingual Contexts: Issues and Challenges", en IRRODL, 12, 6.

Oliver, N. (2015). "El móvil muda la piel”, en Diario El País, 23 de agosto de 2015, disponible en: http://goo.gl/j4KB9S, consultado el 9 de septiembre de 2015.

O'Reilly, T. (2007). What is Web 2.0: "Design Patterns and Business Models for the Next Generation of Software", en Communications \& Strategie, 1: 17.

Pareja, A., Calle, C., y Pomposo, L. (2016). "Aprendiendo a hacer presentaciones efectivas en inglés con BusinessApp". En RIED. Revista Iberoamericana de Educación a Distancia, 19, 1: 41-61.

Pascual, $M^{\mathrm{a}}$ P. (2003). "El Blended learning reduce el ahorro de la formación on-line pero gana en calidad", en Educaweb, 69, 6 de octubre de 2003, disponible en http://goo.gl/bkXCri, consultado el 22 de julio de 2015.

Perona, J.J. y Barbeito, M. (2007). "Modalidades educativas de la radio en la era digital", en ICONO 14, 9.

Precel, K.; Eshet-Alkalai, E.; Alberton, Y. (2009). "Pedagogical and Design Aspects of a Blended Learning Course", en IRRODL, 10, 2.

Perraton, H. (Ed.). (1993). Distance education for teacher training. London, UK: Routledge Press.

Peters, O. (2000). "Digital Learning Environments: New Possibilities and Opportunities", en IRRODL, $1,1$.

Potter, C. S., y Naidoo, G. (2009). "Evaluating large-scale interactive radio programmes", en Distance Education, 30, 1: 117-142.

Read, T., y Barcena, E. (2016). "Metacognition as scaffolding for the development of listening comprehension in a social MALL App". En RIED. Revista Iberoamericana de Educación a Distancia, 19, 1: 103-120.

Rico, M. M., y Agudo, J. E. (2016). “Aprendizaje móvil de inglés mediante juegos de espías en Educación Secundaria”. En RIED. Revista Iberoamericana de Educación a Distancia, 19, 1: 121-139.

Rumble, G. (1997). The costs and economics of open and distance learning. London: Kogan Page.

Saltrick, S., Honey, M. y Pasnik, S. (2004). Television goes to school: The impact of video on student learning in formal education. In New York, NY: Education Development.

Sandberg, J., Maris, M. y Hoogendoom, P. (2014). "The added value of a gaming context and intelligent adaptation for a mobile learning application for vocabulary learning". In Computers \& Education, 76: 119-130.

Sangrá, A., Vlachopoulos, D. y Cabrera, N. (2012). "Building an inclusive definition of e-learning: An approach to the conceptual framework", en IRRODL, 13, 2.

Santiago, R. (2012). "Una revisión de la taxonomía del aprendizaje y las apps educativas en el contexto del mobilelearning”. En S. Trabaldo (Ed.), 10 años de vivencias en educación virtual. Buenos Aires: Net-Learning 
Simões, J., Redondo, R. D. y Vilas, A. F. (2013). "A social gamification framework for a K-6 learning platform", en Computers in Human Behavior, 29, 2:, 345-353.

Shin, W., y Kang, M. (2015). "The use of a mobile learning management system at an online university and its effect on learning satisfaction and achievement". En The International Review Of Research In Open And Distributed Learning, 16, 3.

Snowden, B.L. y Daniel, J.S. (1980). "The economics and management of small postsecondary distance education systems", en Distance Education, 1, 1: 68-91.

Torres Mancera, D.; Gago Saldaña, D. (2014). "Los MOOCs y su papel en la creación de comunidades de aprendizaje y participación". En RIED. Revista Iberoamericana de Educación a Distancia, 17, 1: 13-34.

Tovar, E., y Lesko, I. (2014). "Analysis of successful modes for the implementation and use of OpenCourseWare (OCW) \& Open Educational Resources (OER) in Higher Education. The virtual mobility case". En RIED. Revista Iberoamericana de Educación a Distancia, 17, 1: 131-148.

Vázquez, E.; Fombona, J. y Fernández, A. (2013). "Virtual attendance: Analysis of an audiovisual over IP system for distance learning in the Spanish Open University (UNED)", en IRRODL, 14, 3.

Verdú, C.A. (1998). La demanda de educación superior en España: 1977-1994. Madrid: Ministerio de Educación.

Veeramani, R. y Bradley, S. (2008). UW-Madison online-learning study: Insights regarding undergraduate preference for lecture capture. Wisconsin: University of Wisconsin-Madison, E-Business institute, disponible en: http://goo.gl/SVUoOe, consultado el 25 de julio de 2015.

VitalSource. (2014). "Fourth annual Wakefield survey cites cost of college, technology innovation as top trends in higher education", disponible en: http://goo.gl/RBFpHi, consultado el 12 de junio de 2015 .

Wagner, L. (1977). "The economics of the Open University revisited", en Higher Education, 6: 359-381.

Wakefield, A. B., Carlisle, C., Hall, A. G., y Attree M. J. (2008). "The expectations and experiences of blended learning approaches to patient safety education", en Nurse Education in Practice, 8, 1: 54-61.

Watson, W. R., y Watson, S. L. (2007). "An argument for clarity: What are learning management systems, what are they not, and what should they become?", en Tech Trends, 51, 2: 28-34.

Wedemeyer, C. A. (1981). Learning at the back door. Reflections on non-traditional learning in the lifespan. Madison: The University of Wisconsin Press.

Wesch, M. (2009). From Knowledgable to Knowledge-able: Learning in New Media Environments, en The Academic Commons, disponible en: http://goo.gl/4SwyU3, consultado el 22 de julio de 2015 .

Westera, W. (2012). "The eventful genesis of educational media", en Education and Information Technologies, 17, 3: 345-360.

Woo, K., Gosper, M., McNeill, M., Preston, G., Green, D., y Phillips, R. (2008). "Web-based lecture technologies: Blurring the boundaries between face-to-face and distance learning", en ALT-J, Research in Learning Technology, 16, 2: 81-93 